\title{
NICOLE-CLAUDE MATHIEU: HACÍA UNA ANATOMÍA DE LAS CLASES DE SEXO ${ }^{1^{*}}$
}

\author{
Jules Falquet \\ Universidad Paris Diderot
}

Es difícil presentar en pocas páginas la obra de una intelectual de la talla de NicoleClaude Mathieu, una de las pensadoras más estimulantes del movimiento feminista francés de los setenta. Pero frente a la actual embestida de una vulgata "de género" poco teorizada y mal comprendida y a la creciente confusión sobre lo que es o debería ser el feminismo, esta presentación es hoy más necesaria que nunca. Hay que decirlo: los trabajos de Mathieu deben ser leídos y releídos, particularmente se recomiendan a las nuevas generaciones, ya que son incisivos, pioneros y bastante esclarecedores. Recuerdo de una época y de una ética en las̄ que primaba la calidad sobre la cantidad bibliométrica. Sus textos, relativamente pocos pero muy densos y a menudo largos, son un ejemplo de rigor intelectual. Su claridad y audacia teórica provocan ese salto cualitativo del pensamiento, tan necesario para ayudarnos a encontrar, por fin, puertas por las que salir de las relaciones sociales de poder.

Antes de empezar uno de los viajes posibles por su obra, conviene establecer algunos puntos de referencia para orientar la travesía.

1. Una primera versión de este texto se publicó en francés en la revista Les Cahiers du Genre, n50, 2011 : 193-217. 193-217. Esta traducción al español es de Natalia Santiesteban. Agradezco a Nasima Moujoud por nuestras largas discusiones en torno a este texto. 


\section{TRES RECORDATORIOS PARA UNA TRAVESÍA}

Recordemos, en primer lugar, que Nicole Claude Mathieu posee un doble arraigo disciplinar, en antropología y sociología, que hace de ella una persona con capacidades perceptivas particularmente agudas, una especie de "doble espíritu", si podemos transponer así la concepción que sobre las (los) berdaches tienen algunas poblaciones indígenas de América del Norte. Dicho de otro modo, según su cómplice Paola Tabet, Nicole-Claude Mathieu es una mujer excepcionalmente "bien armada" pues utiliza herramientas de ambas disciplinas, lo que le permite desarrollar análisis con perspectivas globales que incluyen simultáneamente a las sociedades llamadas "no occidentales" y “occidentales", sin establecer ni rupturas a priori, ni primacías.

Pocas veces adoptada, esta perspectiva, cuando se utiliza, a menudo se emplea mal y, sin embargo, Mathieu logra sacar de ella el mayor provecho. En primer lugar, Mathieu no ignora lo que pasa fuera de occidente. Su voluntad de ampliar su horizonte es tanto más interesante que es anterior al periodo de mundialización triunfante y su presión para pensar el mundo como una "aldea global", y con sus facilidades tecnológicas para acceder a la información. Mathieu tampoco establece paralelismos u oposiciones injustificados y menos aún, perspectivas "evolucionistas". No hace caso omiso de las relaciones de poder Sur-Norte: "Que la antropología sea la hija del imperialismo occidental, no sólo históricamente sino en su misma ideología, transmitida en gran parte a través de sus descripciones 'científicas' es, hoy, algo que ya prácticamente se da por sentado" (Mathieu, 1985: 132). Mathieu es aun más circunspecta ante ciertos impulsos “justicieros": "Hoy en día, muchos etnólogos-optan abiertamente por la 'defensa' (y no sólo la ilustración) de las culturas minoritarias: denuncia del imperialismo, de los genocidios, del neocolonialismo o la colonización interior de ciertas minorías por las nuevas (o antiguas) culturas nacionales, denuncia del 'etnocidio' -enfatizando (algo que en parte ya hacía la etnología tradicional) los valores, las organizaciones sociopolíticas y/o las racionalidades económicas consideradas mejores que las nuestras y producidas por algunas de esas culturas que, por 'nuestra' culpa, están en vía de extinción" (Mathieu, 1985:133). Volveremos sobre este punto.

Recordemos también, en segundo lugar, que Mathieu fue una de las fundadoras y principal pensadora de una de las perspectivas teóricas más estimulantes de finales del siglo XX, el feminismo materialista $y$, en particular, de su rama francófona ${ }^{2}$, fruto de una práctica política colectiva mucho más vasta, que cristalizó en Francia en torno a

2. Una de las principales características de esta corriente es afirmar que las mujeres no son una categoría biológica sino una clase social definida por relaciones sociales estructurales de sexo, histórica y geográficamente variables, organizadas alrededor de la apropiación individual y colectiva de la clase de las mujeres por la de los hombres, a través de lo que Colette Guillaumin (1978) ha denominado las relaciones sociales estructurales de sexaje. Esas relaciones están sólidamente apoyadas en lo que llamó la ideología de la Naturaleza, que también sostiene las relaciones sociales estructurales de "raza". 
la revista Questions Féministes que co-fundó y en la que participó hasta la ruptura en 1980. Su definición del feminismo es muy política: "Le daré [...] a la palabra "feminismo" el sentido corriente y mínimo de: análisis hecho por mujeres (es decir, a partir de la experiencia minoritaria) de los mecanismos de la opresión de las mujeres en tanto que grupo o clase, por parte de los hombres en tanto que grupo o clase, en diversas sociedades, y la voluntad de actuar en pro de su abolición. De hecho, considero no tener que exponer aquí los debates políticos internos a los movimientos de mujeres en lo que respecta a las definiciones o a las tácticas pero es útil señalar, desde ahora, que las divergencias entre políticas "feministas" son las mismas en todos los países, sean o no desarrollados, sean o no capitalistas" (Mathieu, 985a:172).

Por tanto, no hay un "sujeto" único ni esencializado del feminismo, sino un conjunto de posiciones socio-políticas lo que, con Colette Guillaumin (1981) y Danièle Juteau (1981), Mathieu llama la experiencia minoritaria y a partir de las cuales se elaboran análisis y se llevan a cabo luchas que no son, en ningún caso, monolíticas. Lejos de toda "sororidad universal", Mathieu subraya que la clase de las mujeres y sus organizaciones están atravesadas por conflictos que son producto de dinámicas y de luchas, tanto en el seno de la clase de las mujeres como en su exterior, para definir las orientaciones del movimiento. Por lo tanto, no se trata de oposiciones mecánicas entre Norte y Sur ni entre capitalismo y socialismo. Para las feministas materialistas el problema no es de las buenas intenciones, lo que hay que hacer es combatir el identitarismo y el naturalismo. No se trata de defender a todas las mujeres o a cualquier mujer, ni de obtener la igualdad o la paridad, ni tampoco de hacer la revolución en un solo hogar o en un solo país. El objetivo es bastante más radical y sobre todo, radicalmente diferente: como lo escribió Monique Wittig, a quien Mathieu (1991) cita en el epígrafe de L'Anatomie politique, "cada palabra debe cribarse cuidadosamente" para acabar con la ideología de "la diferencia de los sexos" y simultáneamente, con las "relaciones sociales estructurales de sexo"3 y la división sexual del trabajo que construyen a las personas como mujeres u hombres.

Y recordemos, en tercer lugar, que el trabajo de Mathieu es relativamente poco conocido, aunque contiene reflexiones fundadoras que renuevan totalmente el pensamiento, y pese a que fuera a menudo, la primera en discutir con seriedad los trabajos de algunos "grandes hombres"4 sobre las relaciones sociales estructurales de sexo. Mathieu debatió metódicamente los trabajos de Bernard Saladin d'Anglure (sobre el sentido de que existiera un "tercer sexo" en las poblaciones Inuit y de Maurice Godelier (su teoría sobre

3. Hemos decidido traducir el concepto central del feminismo materialista, "rapports sociaux de sexe", por "relaciones sociales estructurales de sexo", que crean y oponen la clase social de las mujeres a la clase social de los hombres. Hemos hecho esa opción para no confundirlo con las "relaciones sociales cotidianas entre hembras y varones".

4. Nos hacemos eco aquí del concepto antropológico de "big man" desarrollado precisamente por Godelier, a quien Mathieu critica con agudeza. 
el consentimiento de las mujeres a su dominación), y también los de Pierre Bourdieu (su "descubrimiento" de la dominación masculina y su concepto de violencia simbólica) y-Claude Lévi-Strauss (su interpretación de la división sexual del trabajo y el carácter parcial de sus resultados sobre "el intercambio de mujeres" debido a su focalización reduccionista sobre las sociedades patrilineales y virilocales). Así mismo, Mathieu (1994) fue una de las primeras en Francia en reaccionar ante la propuesta de "turbar el género" 5 de Judith Butler.

Cabe destacar que su trabajo no es desconocido para quienes se han especializado en antropología o en sociología. Su primer artículo, una contribución al séptimo congreso mundial de sociología, fue publicado en francés e inglés en numerosas revistas, entre ellas, el International Journal of Sociology. En el transcurso de su carrera, Mathieu escribió principalmente en l'Homme, el Bulletin de l'association française des anthropologues y el Journal des Anthropologues. También publicó en las Presses Universitaires de France, en las ediciones de l'Ecole des Hautes Etudes en Sciences Sociales y en la Maison des Sciences de l'Homme, además de haber redactado voces para varios diccionarios. En el campo feminista, Mathieu fue cofundadora de la revista Questions Féministes y hasta 1989, sus principales trabajos fueron rápidamente publicados en inglés (no menos de seis en la revista Feminist Issues). Por lo demás, Mathieu ha sido traducida a siete lenguas (español ${ }^{6}$, inglés, alemán, japonés, serbo-croata, italiano y griego) y recibió el título de Doctora honoris causa en ciencias sociales por la Universidad de Laval (Québec).

A pesar del carácter innovador de su producción teórica, hay que subrayar que sus análisis siguen siendo ampliamente ignorados o tergiversados, leídos a medias, en Francia, y también en la academia anglófona, incluyendo a quienes se reivindican de la French Theory y del "French Feminism"7. Es irónico ver cómo debates centrales que ya habían abordado las materialistas francófonas y a los que Mathieu había aportado respuestas completas y rigurosas, vuelven de los Estados Unidos, simplificados y debilitados por una o incluso dos traducciones (del francés al inglés y viceversa). Esta realidad

5. El título del libro de Butler es Gender Trouble, traducido como El Género en disputa, y conlleva la idea de introducir confusión en el género. Mathieu, al contrario, quiere dilucidar los fundamentos de la opresión de las mujeres.

6. Es el caso de su artículo "¿Identidad sexual / sexuada/de sexo? Tres modos d e conceptualización de la relación entre sexo y género ", pp. 130-175 en : Curiel, Ochy \& Falquet, Jules (compiladoras), El Patriarcadao al desnudo. Tres feministas materialistas. Buenos Aires, Brecha lesbica.

7. Sobre la invención en los departamentos de literatura de las universidades estadounidenses de un French Feminism particularmente alejado de lo que son realmente las teorías feministas en Francia, puede consultarse en especial Christine Delphy (1996), "Linvention du "French feminism" : une démarche essentielle", Nouvelles Questions Féministes, 17(1): 15-58 y Stevi Jackson (1996), "Récents débats sur l'hétérosexualité : une approche féministe matérialiste”, Nouvelles Questions Féministes, 17 (3): 5-26. 
interpela tanto a las lógicas científicas de la antropología como de la sociología, como a los mecanismos de difusión, transmisión y discusión de las teorías feministas. También refleja lógicas políticas: los análisis potencialmente más peligrosos para el orden existente suelen ser invisibilizados. En todo caso, los trabajos de Mathieu constituyen una base históricamente pionera y particularmente sólida para teorizar las relaciones sociales estructurales de sexo y para continuar pensando sobre esa cuestión.

\section{UNA EPISTEMOLOGÍA PIONERA}

\subsection{Las relaciones sociales estructurales de sexo: antes y más allá del concepto de género}

La epistemología es uno de los temas que más trabajó Mathieu y lo hizo de forma pionera sobre, al menos, tres problemáticas. Su primer artículo, "Notas para una definición sociológica de las categorías de sexo" (Mathieu 1971), coincide con el reaparecer del feminismo francés en los años 70. Es una guía programática que merece figurar como ejemplo de sociología aplicada a las relaciones sociales estructurales de sexo y como ejemplo de lo que el feminismo puede aportar a la sociología. En este trabajo, Mathieu recuerda que la "clase" o "categoría socio-profesional" se ha vuelto una variable sociológicamente reconocida al haber sido históricamente desnaturalizada a través de las luchas colectivas. Establece un paralelo con la variable "edad", aún frecuentemente naturalizada pero que, a medida que la 'tercera edad' y la 'juventud' empiezan a plantear problemas sociales, será tratada con mayor rigor científica. Por último, analiza el trato que se le da a la variable "sexo". Mathieu muestra cómo aquellos años, gracias al surgimiento del movimiento de las mujeres-feminista, surge la posibilidad de concebir esta última categoría - una de las más naturalizadas - de manera sociológica y científica. Así mismo insiste en la importancia de conceptualizar a las mujeres (hasta entonces casi invisibles para quienes investigan) $=\mathrm{y}$ también a los hombres (hasta entonces, vistos como la norma, como modelo neutro de la humanidad) como categorías sociales, y subraya la necesidad de estudiar estas categorías de forma relacional y dialéctica.

Segunda e iluminadora propuesta, en su siguiente artículo “ ¿Hombre cultura y mujer naturaleza?", publicado en 1973, Mathieu plantea que "los sexos [son] el producto de una relación social” (Mathieu, 1991: 43). Al afirmar que el sexo, i y no el género i, no tiene nada de biológico, abre a las feministas francófonas un camino diferente al de las anglosajonas, quienes seguían la perspectiva abierta por Margaret Mead (que había mostrado la relatividad cultural de los "roles" de sexo), y por la británica Ann Oakley, que propuso en 1972 agrupar esos "roles de sexo", que como Mead consideraba arbitrarios, en el concepto de "género", para diferenciarlos así de lo que consideraba como "natural", el sexo. 
Mientras que las anglófonas se adentran por ese camino, Mathieu encuentra en el concepto de "relaciones sociales estructurales de sexo" la posibilidad de prescindir tanto del sexo como del género -dos conceptos equívocos- además de tener la ventaja de nombrar claramente las relaciones sociales estructurales $y$, en consecuencia, de plantear la cuestión del poder. Probablemente, las notables cualidades y potencialidades del concepto de relaciones sociales estructurales de sexo explican, por qué el concepto de género se impuso tardía y parcialmente en Francia. En paralelo, si el concepto de relaciones sociales estructurales de sexo se ha exportado relativamente poco, a pesar de sus cualidades, quizás sea porque en inglés, en español o en portugués existe un único término (relaciones sociales) para designar a la vez las relations sociales y los rapports sociaux. Esto lleva a confundir los niveles micro (relaciones o vínculos sociales interindividuales, interacciones interpersonales cotidianas, tangibles, relativamente fácilmente negociables y modificables) y macro (relaciones sociales estructurales "abstractas", que no se pueden "tocar" directamente y que son muy estables. Para modificarlas, no basta con cambios individuales, son necesarias las luchas colectivas). Esto es, tal vez, lo que explica la dificultad de ciertas personas del continente americano, para apropiarse plenamente de la perspectiva feminista materialista francófona.

\subsection{Antropología de las mujeres, antropología feminista y punto de vista situado}

En su vasto trabajo de síntesis sobre mujeres y antropología que realiza en 1985 para la UNESCO, Mathieu aporta otras pistas epistemológicas importantes. Vuelve a trazar los comienzos de la antropología de las mujeres y de la antropología feminista, mostrando sus continuidades y sus profundas diferencias: al igual que en historia o en sociología, ciertamente, hay que empezar por visibilizar a las mujeres. Sin embargo, "agregar" a las mujeres es ampliamente insuficiente si no se estudian dialécticamente las relaciones sociales estructurales entre mujeres y hombres. Tal y como dice retomando a Edholm, Harris y Young (1977) y teniendo siempre en cuenta-ambos lados de la relación antropológica (las sociedades estudiadas y las que estudian las demás), "no se trata tanto de buscar a las mujeres 'detrás de' las formas sociales aparentes, como de entender, en las estructuras sociales estudiadas, el significado de su ausencia. (Añadiré que hay que entenderlo también en las estructuras de las sociedades que producen la etnología)" (Mathieu, 1985: 126).

En este texto, particularmente pedagógico, Mathieu presenta sistemáticamente a los "grandes antropólogos" estructuralistas, funcionalistas y marxistas, acto seguido, los aportes críticos de las antropólogas feministas a esos mismos debates. Este diálogo con los trabajos de sus pares es una constante en Mathieu, que reflexionó mucho sobre lo que ella llama "conciencia"8 y que otras personas, más recientemente, denominan punto de vista

8. En el sentido materialista y colectivo, más que psicológico e individual, aun cuando ella no desestima el análisis de los sueños de las mujeres (Ver, "Quand céder...). 
situado. Según Mathieu, se trata de contribuir a edificar la "ciencia de las oprimidas(os)", anunciada por Wittig, Guillaumin o Juteau. Así, para una cabal comprensión de las relaciones sociales estructurales de sexo, ella recomienda, por supuesto, leer y escuchar a las mujeres que, teniendo una experiencia directa de la dominación, son las mejores conocedoras de sus efectos, algo que no desmentiría bell hooks (1981). No obstante, Mathieu preconiza también una lectura atenta de los hombres: aunque estén marcados de sesgos androcéntricos, en tanto que dominantes, pueden proporcionar una mejor comprensión de los mecanismos que les permiten dominar, una posición no separatista que no desaprobaría Barbara Smith (1983).

\subsection{Androcentrismo y etnocentrismo: la crítica de las sociedades occidentales}

Mucho antes de los debates en torno al sexismo y al racismo que surgieron a raíz de la promulgación de la ley francesa de 2004 sobre símbolos religiosos en la escuela, Mathieu $(1987,1993,1995)$ ofreció en varios artículos agudas reflexiones sobre la posición de la antropología francesa frente a las mutilaciones genitales femeninas, analizando los estrechos vínculos que existen entre etnocentrismo y androcentrismo, así como lo que ambos esconden en las sociedades estudiadas y, especialmente, en las sociedades occidentales.

Así, en el texto bellamente titulado "Femmes de soi, femmes de l'Autre" (Mujeres de sí, Mujeres del Otro), Mathieu (1987) subraya que "a menudo, las mujeres occidentales (etnólogas o no) que insisten en la opresión física, económica y mental de las mujeres en muchas sociedades, son acusadas de entrometerse en los asuntos "internos" de otros grupos o pueblos; son acusadas de etnocentrismo, de imperialismo y hasta de racismo. [... Ahora bien] a) Hay mujeres en las sociedades en cuestión, por ejemplo africanas, que se oponen a las mutilaciones y a la opresión (Thiam, 1978; Modefen, 1982) y no se trata solamente de "valores modernos" [...] b) las feministas denunciaron primero la barbarie de Occidente frente a sus propias mujeres (entre otras, la ablación del clítoris en el siglo XIX y la episiotomía en el siglo XX, la esclavitud sexual, etc.) contrariamente a colonialistas y racistas, que sólo denunciaron las de los otros" (Mathieu, 1987: 608). Tras ésta útil precisión, Mathieu añade: "Disociar la noción de minoritaria(o) de la noción de 'mujer' en las otras culturas permite (por la acusación de etnocentrismo) negar un problema del que las y los etnólogos forman parte en su propia sociedad: el del androcentrismo, debido a las relaciones de poder entre los sexos. [...] En suma, hablar de injerencia en los 'asuntos internos' de otras sociedades, en lo concerniente a los sexos consiste; de hecho, por una parte, en negarse a pensar en nuestros asuntos internos; por otra parte y correlativamente, en continuar disimulando una realidad fundamental de las sociedades estudiadas" (Mathieu, 1987: 610). Finalmente, precisa en otro texto: "Sostengo que las acusaciones de etnocentrismo hechas contra las que insisten en la 
opresión de las mujeres por los hombres en las otras sociedades no son mas que una nueva transformación, cargada de culpa, del mismo etnocentrismo: considerar a las sociedades occidentales como "aparte", bajo el pretexto que oprimen a otras" (Mathieu, 1985: 125).

Actualmente se acusa mucho a las feministas "blancas" de juzgarlo todo por el rasero de valores "occidentales" o "burgueses" y de querer "universalizar" categorías o experiencias muy específicas. Indudablemente, esto se observa claramente en el feminismo liberal —en el que se inscriben las instituciones internacionales y muchas ONGs- y aún se observa demasiado a menudo en las corrientes "socialistas" y "radicales" (que por lo demás distan de estar conformadas únicamente por "blancas", "occidentales" o "burguesas"). Mathieu, sin embargo, se sitúa en otra parte.

En su trabajo no se trata de universalizar, ni de guiar a nadie, sino de pensar en las diferencias y semejanzas entre las sociedades llamadas occidentales y las llamadas no occidentales: "Parece más esclarecedor reconocer que en la mayoría de los casos existe, en cuanto al poder de los hombres sobre las mujeres, el 'viriarcado', una similitud estructural entre nuestras sociedades y otras -más allá de los contenidos específicos. [...] Este carácter de proximidad en cuanto a las relaciones de sexo entre las sociedades occidentales y otras sociedades -particularmente patrilineales, patri-virilocales y fuertemente viriarcales (que representan más del $80 \%$ de las sociedades conocidas y sobre las cuales están basadas la mayoría de las teorizaciones etnológicas)- produce simultáneamente cegueras y empatías entre investigadores y etnólogos" (Mathieu, 1985: 125-6). Gracias a esta manera de proceder Mathieu nos propone sus análisis más apasionantes.

\section{UN ANÁLISIS GLOBAL PERO NO UNIVERSALIZANTE DE LOS VÍNCULOS ENTRE ANATOMÍA Y ECONOMÍA POLÍTICA}

\section{1. “Cuerpos que importan" : la anatomía política}

Tras L'arraisonnement des femmes ${ }^{10}$, obra colectiva que coordina publicándose en 1985, hay que esperar hasta 1991 para que las ediciones Côté-femmes reúnan seis de los principales artículos de Mathieu en lo que fue, hasta poco antes de su muerte, su única obra exclusivamente personal. El título es un programa en sí mismo: L’anatomie politique: catégorisations et idéologies du sexe [La anatomía política: categorizaciones e ideologías del sexo], y constituye una prueba de que para las materialistas, el cuerpo sí importa. De especial importancia es el cuerpo para Mathieu, mucho antes que para Judith Butler

9. Del latin vir = varón. Viriarcado es un concepto acuñado por Mathieu para hacer referencia al poder de los hombres en tanto que personas de sexo masculino, más que como padres o patriarcas. El concepto de patriarcado le parecía insuficiente.

10. El título juega con el doble sentido de la palabra arraisonnement : por un lado, arrestar o reducir (como un barco arrestado por las autoridades portuarias), por otro, hacer volver a la razón, ajuiciar. "Larraisonnement des femmes" podría traducirse como : Ajuiciando/arrestando a las mujeres. 
- y para Mathieu, decir que es socialmente construido es una evidencia. Ella afirma que el cuerpo es político, no solo modificable individualmente, sino que colectivamente modificado, y esto dese hace tiempo y en las más variadas sociedades. La originalidad de Mathieu con relación a quienes le suceden, es que evidencia muy claramente que, a pesar de todas las transformaciones, desviaciones o resistencias basadas en el cuerpo, "en la base y en el punto más bajo de la escala de los géneros, definitivamente hay hembras: sexo social 'mujer"', tal y como concluye en la penúltima página de L'anatomie politique..., (Mathieu, 1991: 266).

Mathieu no tiene, sin embargo nada de victimista: la última página de L’anatomie politique es la foto de una mujer de 90 años que blande las dos armas con las que "capturó a un atracador que quiso robarle en su casa" (Mathieu, 1991: 267). Así las mujeres, incluso en la vulnerabilidad de la vejez, pueden resistir a las agresiones de manera concreta, directa, violenta, y no únicamente simbólica. Entre las posibles herramientas para transformar las relaciones sociales estructurales de sexo, Mathieu nos muestra las armas de fuego y no el bigote.

\subsection{Diversidad de formas de concebir la articulación entre sexo, género y sexualidad}

Para Mathieu, la anatomía (construida) es muy importante. Sin embargo, lo más importante es la diversidad sociocultural e histórica de las interpretaciones que se hacen de esa anatomía, como lo demuestra en su magistral artículo "Identité sexuelle/ sexuée/ de sexe? Trois modes de conceptualisation des rapports entre sexe et genre" [¿Identidad sexual/sexuada/de sexo? Tres modos de conceptualización de la relación entre sexo y género] ${ }^{11}$. En él responde al antropólogo Saladin d’Anglure, quien afirma que la existencia de un "tercer sexo" en la sociedad Inuit invalidaría la construcción binaria de los géneros y los sexos — debilitando así la teoría de la opresión de las mujeres. Mathieu compara ese "descubrimiento" con muchas otras prácticas "desviadas", individuales o colectivas, permanentes u ocasionales, relativas a la sexualidad, al género o al sexo, que un pensamiento occidental un poco ingenuo o etnocéntrico calificaría hoy de queer. Así demuestra que muchas de esas "transgresiones" son en realidad mecanismos institucionalizados de ajuste que no cuestionan de ninguna manera la norma. Demuestra sobre todo, que no existe una única manera de concebir la articulación entre sexo, género y sexualidad (tal y como lo afirma la actual concepción occidental dominante), sino tres:

- Modo I: Identidad "sexual", basada en una conciencia individualista del sexo. Correspondencia homológica entre sexo y género: el género traduce el sexo

\footnotetext{
11. Nicole-Claude Mathieu presentó las bases de este trabajo en México en 1982, en el marco del X congreso mundial de sociología. En 1989 se publicó en Daune-Richard, Hurtig \& Pichevin, y en 1991 en L'anatomie politique.
} 
- Modo II: Identidad "sexuada", basada en una conciencia de grupo. Correspondencia analógica entre sexo y género: el género simboliza el sexo (y viceversa)

- Modo III: Identidad "de sexo", basada en una conciencia de clase. Correspondencia socio-lógica entre sexo y género: el género construye el sexo. (Mathieu, 1991 [1989]: 231).

Así, Mathieu permite pensar tres cosas fundamentales: en primer lugar, que tampoco en este ámbito las divisiones se establecen entre sociedades occidentales y no occidentales, sino en el interior de cada sociedad. Por ejemplo, si el modo II es más característico de las sociedades consideradas tradicionales, también corresponde con el pensamiento de corrientes lésbicas o feministas occidentales (ciertas feministas socialistas en Gran Bretaña y la tendencia "lucha de clases", en Francia). Las lesbianas políticas, a semejanza de Wittig y las feministas materialistas como Tabet, por su parte, adhieren al modo III, al igual que grupos de mujeres en lucha en China o en Sierra Leona. En segundo lugar, que una gran parte de los movimientos homosexuales y lésbicos, pero también queer o trans, están basados en una adhesión inconsciente al modo I, que es el más naturalista (y el más frecuente en las sociedades occidentales). Finalmente, que las transgresiones (reales o supuestas) del sexo, del género o de la sexualidad no resuelven el problema de fondo. Es decir, que las hembras están, de cualquier forma, casi siempre colocadas en lo más bajo de la escala social ${ }^{12}$. Por eso, los movimientos homosexuales de hegemonía masculina no son de ninguna manera los aliados naturales de los movimientos feministas y lésbicos que luchan, en primera instancia, por los intereses de la clase de las mujeres. Ahora bien, el problema de la clase de las mujeres no es tanto la arbitraria definición de los géneros o la obligatoriedad de prácticas sexuales heterosexuales (consecuencias sociales de su apropiación por la clase de los hombres), como su proclamada inferioridad con relación a la clase de los hombres, la obligatoriedad de la maternidad social y, sobre todo, la casi total negación del acceso a los recursos.

\section{3. "Prefiero clarificar la economía política del género [antes] que turbarlo a precio de rebajas.}

Mathieu también escribió sobre la cultura popular "occidental" y publicó en 1994 "Dérives du genre/stabilité des sexes. [Derivas del género/estabilidad de los sexos]”, un artículo sobre Madonna. En un momento de repliegue del movimiento feminista en Francia, critica, en este texto, el postmodernismo que se desarrolla al otro lado del Atlántico, su idealismo, su lenguaje abstruso y especialmente, su proyecto de "confundir/disputar el género" (particularmente los trabajos de Judith Butler, entonces casi desconocida en Francia). De hecho, lo que comienza a regresar a Francia, consagrado por su paso por 12. Mathieu propone, principalmente, el ejemplo de las berdaches de sexo femenino que, pese a que son consideradas socialmente como hombres, pueden ser violadas y a menudo se les juzga como menos dotadas que los berdaches de sexo masculino en lo que a técnica y poderes espirituales se refiere (L'anatomie politique, pp 263-64). 
el establishment universitario norteamericano como French feminism y French theory, y que alimentará en especial al movimiento queer surgido en la década de los noventa, es lo que Mathieu siempre criticó como feminista materialista: un discurso filosóficopsicoanalítico apoyado en Lacan, Derrida y Foucault por el lado masculino, y por el femenino, en la tríada más bien antifeminista Kristeva, Cixous e Irigaray ${ }^{13}$.

En ese artículo, Mathieu se muestra, antes que nada, respetuosa con la artista - una mujer de origen popular - al citar un extracto de una entrevista de Madonna (donde ésta afirma que no conoce nada sobre el movimiento de las mujeres pero que lucha para ser reconocida como un ser humano). Por lo demás, la autora no muestra un entusiasmo delirante por el travestismo de la cantante ni por la bisexualidad y plurirracialidad de sus escenificaciones que, si bien fascinan a parte del mundo universitario, no logran engañar a las chicas jóvenes de barrios populares quienes, efectivamente, observan con atención estos espectáculos para saber "lo que les gusta a los hombres" pero saben pertinentemente que en sus vidas reales, tales fantasías les llevarían a ser inmediatamente etiquetadas como "zorras".

En su análisis, Mathieu no sólo da muestras de su reflexión feminista materialista, sino también de una sólida conciencia de clase: no olvidemos que dedica L’anatomie politique a su abuela "Alice Cartier, obrera a los 13 años". A lo que Mathieu obstinadamente llama, mucho más que a confundir/disputar individualmente el género, es a clarificar colectivamente "la economía política del sexo" ${ }^{14}$, un proyecto que retoma en línea directa del análisis de la "primera" Gayle Rubin ${ }^{15}$.

\section{HERRAMIENTAS Y ARMAS CONTRA LA OPRESIÓN}

\subsection{La conciencia dominada: ¿una "esquizofrenia" constitutiva y potencialmente política?}

Uno de los textos más conocidos de Mathieu es probablemente "Quand céder nest pas consentir" [Cuando ceder no es consentir]. En él analiza magistralmente los

13. Como es sabido sólo la última se reivindica feminista. A propósito de la crítica del "feminismo de la diferencia", puede consultarse el primer número de Questions Féministes.

14. Mathieu tradujo el artículo de 1975 de Rubin “The traffic in women : Notes on the 'political economy' of sex", que se publicó en 1998 bajo el título Léconomie politique du sexe : Transactions sur les femmes et systèmes de sexe/genre, en Les Cahiers du CEDREF (Université Paris 7), $\mathrm{n}^{\circ}$ 7. También tradujo a Gail Pheterson (Le prisme de la prostitution), Paris, L'Harmattan, 2001.

15. A partir de los años 80 , Gayle Rubin se aleja bastante de la perspectiva materialista y deviene una teórica de la sexualidad (de las prácticas sexuales) para quien todas las sexualidades no hegemónicas deberían aliarse frente a la sexualidad heterosexual dominante. Esa posición se aleja mucho de lo que propusieron las materialistas, entre ellas Wittig, para quienes la heterosexualidad no es una práctica sexual sino un sistema político cuyo eje ideológico es "el pensamiento straight". En este caso, el lesbianismo no es una práctica sexual disidente, sino una perspectiva política radical que cuestiona directamente las relaciones sociales estructurales de poder entre la clase de las mujeres y la de los hombres. 
determinantes materiales de la conciencia dominada para rebatir la idea de Godelier según la cual las mujeres "consentirían con su situación" con respecto a su situación. También critica vivamente el término de "dominación", apreciado por l@s mayoritari@s -a quienes favorece- y evitado por 1@s minoritari@s, cuya comprensión nubla. "La palabra 'dominación' distrae la atención hacia aspectos relativamente estáticos, de 'posición superior' (como la montaña que domina al valle, por ejemplo); de 'autoridad' y de 'mayor importancia'. Contrariamente, el término "opresión" implica e insiste en la idea de violencia ejercida, de exceso, de ahogo" (Mathieu, 1985a: 225).

Resaltemos tres puntos importantes de este artículo. Primero: el peso de los determinantes materiales y corporales sobre la conciencia, y más particularmente del agotamiento físico crónico de las mujeres, aliado a la desnutrición generalizada, y que Mathieu es una de las escasas antropóloga y sociólogas en señalar. Segunda evidencia notable, silenciada con demasiada frecuencia: la repartición desigual de la "cultura" según el sexo. Mathieu nos recuerda con fuerza que las mujeres generalmente no tienen acceso a las mismas informaciones sobre "su" cultura (sea el acceso a la alfabetización, a la educación científica o sexual, o a los conocimientos religiosos, filosóficos o esotéricos) que los hombres. Esto constituye una interpelación capital ante el crecimiento de los nacionalismos, tanto mayoritarios como minoritarios: Mathieu nos permite pensar que las mujeres no están obligadas a alinearse en un campo u otro, máxime cuando "sus" propios hombres las excluyen generalmente de la definición, del disfrute y de la oportunidad de encarnar la versión más legítima de "su" cultura. Tercera enseñanza útil: Mathieu explica, en el caso de las mujeres, el fenómeno del estar escindidas entre valores diferentes, incluso opuestos. Es un fenómeno que ha sido analizado más por y para las personas racializadas: es lo que Gloria Anzaldúa (1987) llama la "conciencia de la Mestiza", bell hooks (1981) y Patricia Hill Collins (1990) el "privilegio epistémico" de las mujeres Negras que son "outsiders within", Paul Gilroy (1993) la "doble conciencia"- y que yo llamo "la esquizofrenia legítima y política de los grupos oprimidos". Mathieu propone el ejemplo de la diferencia en las expectativas sociales hacia las mujeres y los hombres en ciertas ceremonias en las que se llevan a cabo escarificaciones ${ }^{16} \mathrm{y}$ en las que debe mostrarse valentía frente al dolor (ideal "cultural"). En algunas de esas ceremonias, los hombres deben dar prueba de una resistencia máxima, mientras que las mujeres deben autolimitarse (y ostentar luego menos escarificaciones), pues si muestran "demasiada" valentía, serán mal consideradas como futuras esposas (ya que golpearlas no tendría efecto sobre ellas).

En otras palabras, las mujeres deben adherir a los valores dominantes de "sus" sociedades, pero simultáneamente deben quedarse "en su sitio", encarnando lo contrario de lo que es considerado como masculino (es decir, encarnando el contrario del ideal cultural de "su"

16. En las que se corta la piel de las personas con navajas u otros instrumentos, para producir cicatrices permanentes que son socialmente apreciadas. 
sociedad). Ser $y$ no ser: el problema es complejo y con él se enfrentan tanto las mujeres como los demás grupos minorizados. Para resolverlo, muchos grupos minoritarios desarrollan una especie de esquizofrenia, emocionalmente agotadora e incluso patógena, pero que puede convertirse en una lucidez y fuerza considerables.

\subsection{Bourdieu: ira y risa para con los "grandes hombres"}

Quince años después de que Mathieu refutara espléndidamente el concepto de dominación en "Quand céder n’est pas censentir", artículo que Pierre Bourdieu parece ignorar totalmente, éste publica un opúsculo, La dominación masculina, que obtiene un éxito rotundo. Si bien la notoriedad de ese libro se explica fácilmente por su brevedad, su inocuidad y por la fama de su autor, se trata -como muestra Mathieu (1999) en un artículo publicado en Les Temps Modernes- de una obra de insostenible ligereza científica. Mathieu será sin embargo, una de las pocas en atreverse a criticar, seriamente pero con un humor corrosivo, al gran hombre.

Sabemos que, además de la debilidad del concepto de dominación, es sobre todo el concepto de "violencia simbólica", desarrollado por Bourdieu, el centro del problema. Como socióloga informada, Mathieu debe subrayar el inmenso peso de la violencia real y material ejercida por los hombres contra las mujeres, analizada desde hace varias décadas por las feministas y que la encuesta nacional oficial ENVEFF ${ }^{17}$ confirmará en 2002. Mientras que Bourdieu propone al amor como remedio posible a la dominación masculina (con una ingenuidad y una ignorancia asombrosas en un científico de su edad y de su rango) la encuesta ENVEFF también mostrará que la violencia contra las mujeres la ejercen sobre todo dentro de la familia las personas que supuestamente las aman.

Finalmente, traigamos a colación las ocho críticas dirigidas por Mathieu al "alumno Bourdieu", que sería suspendido inmediatamente si se le aplicaran a su trabajo los criterios científicos clásicos: 1) No citar autores importantes que han trabajado sobre el tema (entre otras Françoise Héritier, su colega en el Collège de France, pero también Delphy, Guillaumin y Tabet), 2) Referencia superficial a ciertas autoras importantes, deformando su teoría (Lévi-Strauss), 3) Cita de ciertas autoras con alusiones erróneas a sus teorizaciones, o a propósito de un detalle y sin referencia teórica (Gayle Rubin, Gail Pheterson) 4) Alusión, sin citar a su autora, a ciertas teorías que tratan directamente del tema (Godelier), 5) Recurrir a una profusión superficial de referencias (anglosajonas principalmente), 6) Probable recurso a notas de segunda mano (confundiendo a Jeanne Favret-Saada con Mathieu), 7) Uso de un título abusivo y engañoso para su obra ("la parte simbólica incorporada de la dominación masculina” hubiera sido más exacto) $\mathrm{y}$, por último, 8) "el trabajo del candidato carece de rigor técnico, metodológico, y deontológico. Peca por pensamiento, por acción, por omisión y por distorsión. En su

17. Enquête Nationale sur les Violences Envers les Femmes en France [Encuesta Nacional sobre las violencias hacia las mujeres en Francia]. 
totalidad, debe ser interpretado como una negativa a dar pie a la confrontación entre diferentes análisis, lo que da a su tesis un estatus de aserción y no de demostración" (Mathieu, 1999: 63). Y para meditar, cabe añadir esta observación, que podría aplicarse a muchos otros trabajos: "Podemos preguntarnos si no se trata [...] de una demostración particularmente evidente de la dominación masculina, que redobla la opresión de las mujeres al suprimir o distorsionar sus experiencias y sus análisis" (Mathieu, 1999: 63-4).

\section{LEJOS DEL VIRIARCADO Y DEL MATRIARCADO: CUANDO LAS HIJAS SON UNA BENDICIÓN}

En los años 2000, Mathieu publicó principalmente artículos teóricos de síntesis y textos para diccionarios especializados. Entre ellos, señalemos una breve crítica del concepto de "matriarcado", particularmente útil para poner un punto final a las interminables discusiones sobre el "poder oculto" de las mujeres y el fantasma de la inversión de los roles. En ese trabajo, Mathieu lamenta la instrumentalización, con fines ideológicos o incluso turísticos, de un conjunto de prácticas supuestamente "matriarcales" en algunas sociedades. En particular, vuelve sobre dos puntos clave: el primero, que no se conocen sociedades "matriarcales" en el sentido en que, en ellas, los hombres serían tratados como lo son las mujeres en las sociedades viriarcales. En ningún lugar ni en ninguna época ha existido simetría en la brutalidad de la opresión. El segundo, que esa simetría no puede de ninguna manera representar el pasado glorioso, ni el futuro radiante que reivindica el feminismo materialista: éste jamás se ha propuesto invertir la opresión sino más bien, cabalmente, abolir las relaciones sociales estructurales de sexo viriarcales.

Ese texto también constituye una especie de introducción a la gran obra a la que Mathieu (2007) se dedicó durante la última década y que coeditó con la joven antropóloga Martine Gestin: Une maison sans fille est une maison morte. La personne et le genre dans les sociétés matrilinéaires et/ou uxorilocales [Una casa sin hija es una casa muerta. La persona y el género en las sociedades matrilineales y/o uxorilocales]. Muy esperada, esa obra de más de 500 páginas sienta un precedente importantísimo: por sorprendente que pueda parecer, nadie antes de Mathieu había trabajado sistemáticamente y de manera comparativa ${ }^{18}$ sobre las sociedades uxorilocales ${ }^{19}$. Por cierto, éstas sólo representan aproximadamente el 7\% de las 565 sociedades catalogadas en el World ethnographic sample de 1957. No obstante, constituyen hasta el 20\% de las sociedades en África, un cuarto en el Pacífico y hasta un tercio en América del Norte. ¿Qué habría motivado semejante desinterés? Observaremos que, sin ser "matriarcados", esas sociedades son menos desiguales que las otras, desde el punto de vista de las relaciones sociales estructurales de sexo; son históricamente anteriores al modo de producción capitalista y se sitúan fuera del mundo occidental (al que tanto le complace creer que encabeza los "avances" en la igualdad entre

18. Exceptuando los trabajos de Alice Schegel (1972) sobre 66 sociedades matrilineales

19. Sociedades en las que la pareja recién casada se instala con la familia de la esposa o cerca de ella. 
los sexos).

Esta obra reúne a quince autor@s que analizan catorce sociedades extremadamente diferentes (que presentan diversos grados de inclusión en la sociedad global así como de matrilinealidad y/o uxorilocalidad, y que poseen un peso demográfico variado ${ }^{20}$. Buena parte (la mitad) aún existen hoy en día y una de ellas, la sociedad Ngada de Indonesia, es uno de los raros casos conocidos de paso de la patrilinealidad virilocal a la matrilinealidad uxorilocal (lo inverso es generalmente presentado como la evolución "natural" de las sociedades). Las sociedades matrilineales y sobre todo uxorilocales son particularmente interesantes por al menos cuatro razones:

- la matrilinealidad inscribe estructuralmente la producción de hijas en la continuidad del grupo que funda la identidad individual y social

- el poder masculino allí es menos fuerte que en las sociedades patrilineales

- cuando coexisten, la matrilocalidad refuerza la matrilinealidad

- finalmente, la uxori-matrilocalidad reforzaría la conciencia de grupo sexuado entre las mujeres gracias a la estabilidad territorial que genera para ellas

Las consecuencias teóricas de esta obra son apasionantes e imposibles de resumir aquí. Sin embargo, queremos presentar rápidamente los cuatro campos de reflexión que abre.

En primer lugar, a propósito de los fundamentos de la opresión. Si bien las religiones y los mitos fundamentan generalmente el poder de los hombres sobre las mujeres, encontramos aquí poblaciones cristianizadas o islamizadas donde la suerte de las mujeres parece bastante envidiable. Con mayor profundidad, es particularmente revelador el caso (extremadamente raro) en el que las mujeres tienen el monopolio de lo "religioso". Por ejemplo en la sociedad Kavalan (Taïwan), durante mucho tiempo, los hombres han sido rigurosamente excluidos del contacto con los poderes del más allá y las prácticas chamánicas se han reservado estrictamente a las mujeres. En sus mitos de origen o en los de poblaciones próximas, dos hermanos matan a su padre ${ }^{21}$. Aquí, el homicidio del padre no es el pilar de la exogamia que funda el vínculo social, sino "la negación de la transmisión del poder y de los objetos materiales o de las riquezas entre un padre y sus hijos" (Mathieu \& Gestin, 2007: 394). De esta forma, "se les impide a los hombres como grupo la apropiación y acumulación de riquezas. Además, los hombres como grupo no constituyen una unidad de producción, ni una unidad de consumo. Ellos no cazan ni van de pesca colectivamente como clases de edad" (Mathieu \& Gestin, 2007: 395). Estas observaciones, ciertamente, son claves para seguir reflexionando sobre los medios concretos de creación de impotencia en determinado grupo social.

20. La sociedad Minangkabau, en Sumatra, cuenta aproximadamente con tres millones de personas.

21. En otro caso, un padre holgazán mata a su hijo. 
En segundo lugar, el libro abre un campo de reflexión sobre los móviles de la opresión y más particularmente sobre los vínculos entre opresión y cantidad de trabajo que socialmente se espera de personas y grupos. Varios artículos muestran, de hecho, que hasta en sociedades en las que el estatus de las mujeres es mucho mejor que en otras, la cantidad de trabajo que deben realizar es considerable. Tal es por ejemplo el caso en la sociedad $\mathrm{Na}$ (Mosuo), en China, donde la ociosidad de los hombres es notoria. Tenemos allí pues, una pista más bien contra-intuitiva, que demanda mayor exploración.

La tercera pista que nos ofrece el libro es la del replanteamiento de las "teorías fundamentales" de la antropología, en este caso la del "el intercambio de las mujeres", tema abordado en de la larga conclusión escrita por Martine Gestin. En primer lugar, contrariamente a lo que había pensado Lévi-Strauss, ese “intercambio" dista de ser universal. Chantal Collard (2000) ya había demostrado que las mujeres podían ser unas "sujetas activas del intercambio", incluso en sociedades patrilineales. Sin embargo, las sociedades analizadas en Une maison sans fille abren un horizonte todavía más vasto. En ellas, las mujeres pueden -con mayor razón- ser sujetas activas del intercambio, pero sobre todo, el rol sociocultural fundamental que la antropología atribuyó al intercambio matrimonial, merece ser relativizado. Así, en la sociedad Kavalan, la circulación de riquezas en ocasión de los intercambios matrimoniales juega un papel menor, comparado con los intercambios anuales de alimentos durante la fiesta de iniciación chamánica de las mujeres. Lo que es significativo para las relaciones sociales estructurales de sexo, es que son las mujeres que son las operadoras de esas dos redes de intercambio.

Finalmente, aunque no sea uno de sus temas de discusión central, Une maison sans fille proyecta nuevas luces sobre la articulación de las relaciones sociales estructurales de sexo, "raza” y clase. Entre las preguntas interesantes se encuentra la de ¿qué pasa en las uniones "mixtas" entre mujeres de sociedades matrilineales y sobre todo uxorilocales (a menudo dominadas en la sociedad global) con hombres de sociedades patrilineales y virilocales (a menudo dominantes)? Otra pregunta candente es la de la co-extensividad (o no) de las relaciones sociales estructurales de sexo y de clase. Así, en la sociedad Minangkabau de Sumatra, la casta social esclavizada, endógama, practica el intercambio matrimonial simétrico. Las personas "libres", por su parte, practican eventualmente uniones asimétricas (entre un hombre de rango superior y una mujer de linaje inferior) aunque, en ese caso, "para pagar el precio de un prometido de mayor posición, las mujeres de rango medio entran en una relación de servicio con las madres o las hermanas de su marido de rango superior, constituyendo así una reserva de mano de obra (generalmente a la manera de la aparcería). [Aquí sin embargo] la matrilinealidad y la matrilocalidad, que ubican claramente a las mujeres del lado de las riquezas, impiden que se imponga, en el intercambio matrimonial, la coextensión de las relaciones de género y de las relaciones 
de clase" (Mathieu \& Gestin, 2007: 461). Tal configuración invita a reflexionar.

Al final de esta travesía -parcial y demasiado rápida- no podemos más que constatar que el trabajo pionero, apasionante y revolucionario de Nicole-Claude Mathieu nos alimentará política e intelectualmente durante mucho tiempo y que puede abrirnos las puertas hacia otro imaginario feminista. En 2014, poco antes de su muerte, se re-editó L'Anatomie Politique y sobre todo se publicó L'Anatomie Politique 2. Usage, déréliction et résillience des femmes, que reúne el resto de sus trabajos aún dispersos. Así antes de irse Nicole-Claude Mathieu tuvo a bien dejarnos, de forma muy concreta, las herramientas y armas para desarrollar una verdadera ciencia y conciencia de las oprimidas desde su punto de vista, iy para ellas! 


\section{REFERENCIAS BIBLIOGRÁFICAS}

Anzaldúa, Gloria (1999) [1987] Borderlands: La Frontera, The New Mestiza. San Francisco: Aunt Lute.

Collard, Chantal (2000) «Femmes échangées, femmes échangistes ». L’Homme 154-155, abril-septiembre, pp 101-116.

Gilroy, Paul (2003) L’Atlantique noir. Modernité et double conscience. París: Kargo (primera edición en inglés, 1993).

Guillaumin, Colette (1978) "Pratique du pouvoir et idée de Nature" (I). Lappropriation des femmes. (II) Le discours de la Nature. Questions Féministes, 2-3

_ (1981) «Femmes et théories de la société : remarques sur les effets théoriques de la colère des opprimées ». Sociologie et société, 13 (2), pp. 19-31.

_ (1992) Sexe, Race et Pratique du pouvoir, l'idée de Nature. Paris : Côté Femmes.

Hill Collins, Patricia (1990) Black Feminist Tought: Knowledge, Consciousness and the Politics of Empowerment. London: Harper Collins.

Hooks, Bell (1981) Ain't I a woman? Black women and feminism. Boston: South End Press.

Juteau, Danielle (1981), «Visions partielles, visions partiales : visions des minoritaires en sociologie ». Sociologie et société 13 (2), pp. 33-47.

Mathieu, Nicole-Claude (1971) “Notes pour une définition sociologique des catégories de sexe ". Epistémologie sociologique 11, pp. 19-39. Re-editado en L’Anatomie politique..., pp. 17-41.

_ (1973) “Homme-culture et femme-nature ? ”. L'homme, revue française d'anthropologie XIII (3), pp. 101-113. Re-editado en L’Anatomie politique... , pp. 43-61.

_ (1985) "Critiques épistémologiques de la problématique des sexes dans le discours ethno-anthropologique”. Rapport pour l'UNESCO, Réunion internationale d’experts : “ Réflexion sur la problématique féminine dans la recherche et l'enseignement supérieur ", Lisbonne, 17-20 sept. 1985. Paris, UNESCO, SHS-85/CONF. 612/6, 61 p. dactyl. Publicado en L'Anatomie politique..., pp. 75-127.

_ (1985a) “Quand céder n’est pas consentir. Des déterminants matériels et psychiques de la conscience dominée des femmes, et de quelques-unes de leurs interprétations en ethnologie ”. En N.-C. Mathieu (ed.) L’anatomie politique..., pp. 131- 225.

Mathieu, Nicole-Claude, ed., (1985b) L'Arraisonnement des femmes. Essais en anthropologie des sexes. Paris: Eds de l'Ecole des Hautes Etudes en Sciences Sociales (EHESS). 
Mathieu, Nicole-Claude (1987) “Femmes du Soi, femmes de l'Autre”. En Vers des sociétés pluriculturelles : études comparatives et situation en France. Actes du Colloque international de l'Association française des Anthropologues (AFA), Paris, 9-11 janvier 1986, pp. 604-614. Paris : Ed. l'ORSTOM.

_ (1989) "Identité sexuelle/sexuée/de sexe ? Trois modes de conceptualisation du rapport entre sexe et genre ". En A.-M. Daune-Richard, M.-C. Hurtig \& M.-F. Pichevin (eds.) Catégorisation de sexe et constructions scientifiques, Aix-en- Provence, Université de Provence, pp. 109-147. Re-editado en L'Anatomie politique..., pp. 227- 266.

_ (1991) L’Anatomie politique. Catégorisations et idéologies du sexe. Paris:Côté-femmes.

_ (1994) "Dérive du genre/stabilité des sexes". En Michel Dion (ed.) Madonna. Erotisme et pouvoir. Paris : Editions Kimé, pp. 54-70.

_ (1999) "Bourdieu ou le pouvoir auto-hypnotique de la domination masculine". Les Temps Modernes, $\mathrm{n}^{\circ}$ 604, mai-juin-juillet, pp. 286-324.

_ (2000) "Un hommage critique à Lévi-Strauss et Freud: Gayle Rubin (1975)”. Journal des Anthropologues, n 82-83, pp. 41-52.

Mathieu, Nicole-Claude (dir) (2007) Une maison sans fille est une maison morte. La personne et le genre en sociétés matrilinéaires et/ou uxorilocales. Paris : Éditions de la Maison des Sciences de l'Homme.

Smith, Barbara. (Ed.) (1983) Home Girls : a Black Feminist Anthology. New York :

Kitchen Table/Women of Color Press.

\section{ANEXO}

\section{Principales artículos y libros de Nicole-Claude Mathieu}

(1971) “Notes pour une définition sociologique des catégories de sexe ". Epistémologie sociologique (11), pp. 19-39. Re-editado en L'Anatomie politique...

(1973) “ Homme-culture et femme-nature ? ". L'homme, revue française d'anthropologie XIII (3), pp. 101-113. Re-editado en L'Anatomie politique...

(1977) “Paternité biologique, maternité sociale... ”. En Andrée Michel (ed.) Femmes, sexisme et sociétés. Paris : Presses Universitaires de France, pp. 39-48. Re-editado en L'Anatomie politique...

(1985) Mathieu, Nicole-Claude (ed.) L'Arraisonnement desfemmes. Essais en anthropologie des sexes. Paris : Editions de l'Ecole des Hautes Etudes en Sciences Sociales. 
(1985) “Quand céder n’est pas consentir. Des déterminants matériels et psychiques de la conscience dominée des femmes, et de quelques-unes de leurs interprétations en ethnologie ". En Mathieu, NC (ed.) Larraisonnement des femmes..., pp. 169-245. Reeditado en L'Anatomie politique...

(1985) "Critiques épistémologiques de la problématique des sexes dans le discours ethno-anthropologique". Rapport pour l'UNESCO, Réunion internationale d'experts : “ Réflexion sur la problématique féminine dans la recherche et l'enseignement supérieur ", Lisbonne, 17-20 sept. 1985. Paris, UNESCO, SHS-85/CONF. 612/6, dactyl. Re-editado en L'Anatomie politique...

(1986) "Sexe, genre et Nature en anthropologie ". Séminaire Limites-Frontières, 21 mars 1986, nº 47, dactyl. Re-editado en Gardey \& Löwy (eds.), Linvention du naturel......

(1987) "Femmes du Soi, femmes de l'Autre". En Vers des sociétés pluriculturelles : études comparatives et situation en France. Actes du Colloque international de l'Association française des Anthropologues (AFA), pp. 604-614. Paris : Ed. de l'ORSTOM.

(1989) "Identité sexuelle/sexuée/de sexe ? Trois modes de conceptualisation du rapport entre sexe et genre ". En A.-M. Daune-Richard, M.-C. Hurtig \& M.-F. Pichevin (eds.) Catégorisation de sexe et constructions scientifiques, pp. 109-147. Aix-en- Provence : Université de Provence. Re-editado en L'Anatomie politique...

(1991) "Tendances actuelles de la recherche en anthropologie des sexes". En Actes du IVème colloque Méga-Tchad (CNRS/ORSTOM, Paris, 14-16 sept. 1988), vol. II : Les relations hommes-femmes dans le bassin du lac Tchad, pp. 13-33. Textes réunis et présentés par Nicole Echard. Paris : Editions de l'ORSTOM.

(1991) L'Anatomie politique. Catégorisations et idéologies du sexe. Paris : Côté-femmes Editions.

(1991) "Les transgressions du sexe et du genre à la lumière de données ethnographiques ". En M.-C. Hurtig, M. Kail \& H. Rouch (eds.) Sexe et Genre. De la hiérarchie entre les sexes. Paris : Presses du CNRS, pp. 69-80.

(1993) “Questions à léco-féminisme ". En Maria Inácia d’Avila \& Naumi de Vasconcelos (eds.) Ecologia, feminismo, desenvolvimento. Rio de Janeiro : EICOS / Universidade Federal do Rio de Janeiro, pp. 128-137.

(1994) “"Origines”, ou mécanismes de l’oppression des femmes?”. En Catherine Fussinger \& Monique Pavillon (eds.) Femmes, le mauvais genre? (Colloque organisé par le groupe “Regards critiques " à l'Université de Lausanne, 5-6 déc. 1990). Lausanne : Faculté des Lettres, Série Histoire et société contemporaines, n 16, pp. 13-30.

(1994) “Dérive du genre/stabilité des sexes”. En Michel Dion (ed.) Madonna. Erotisme et pouvoir. Paris : Editions Kimé, pp 54-70. 
(1995) "Relativisme culturel, excision et violences contre les femmes ". Sexe et race. Discours et formes nouvelles d'exclusion du XIXe au XXe siècle, 1994-tome 9 (CERIC / Université Paris 7), pp. 87-102.

(1999) "Bourdieu ou le pouvoir auto-hypnotique de la domination masculine". Les Temps Modernes, $\mathrm{n}^{\circ}$ 604, mai-juin-juillet, pp. 286-324.

(2000) "Les sexes et la "nature" chez les ethnologues et les ethnologisés (rappel historique) ”. En Delphine Gardey \& Ilana Löwy (eds.) L'Invention du naturel. Les sciences et la fabrication du féminin et du masculin. Paris : Editions des archives contemporaines, pp. 109-124.

(2000) “Anthropologie et "homosexualités" ". En Martine Gross (ed.) Homoparentalités: Etat des lieux. Parentés et différence des sexes. Paris : ESF éditeur. (La vie de l’enfant), pp. 89-94.

(2000) “Sexe et genre”. En Helena Hirata, Françoise Laborie, Hélène Le Doaré \& Danièle Senotier (eds.) Dictionnaire critique du féminisme. Paris: PUF, pp. 191-200.

(2000) "Un hommage critique à Lévi-Strauss et Freud: Gayle Rubin (1975)”. Entretien réalisé par C. Quiminal, Journal des Anthropologues (82-83), pp. 41-52.

(2004) “"Matriarcat" ou résistance ? Mythes et réalités ». Revue Espace lesbien, n 4 (Toulouse, Bagdam Espace Édition), pp. 73-84.

(2007) (Mathieu, Nicole-Claude, dir.) Une maison sans fille est une maison morte. La personne et le genre en sociétés matrilinéaires et/ou uxorilocales. Paris : Éditions de la Maison des Sciences de l'Homme.

\section{Traducciones hechas por Nicole-Claude Mathieu :}

Gayle RUBIN, "The traffic in women : Notes on the "political economy" of sex ". En Reiter, Rayna R. Toward an anthropology of women, New York, Monthly Review Press, 1975 : L'économie politique du sexe: Transactions sur les femmes et systèmes de sexe/genre, traduit de l'anglais par Nicole-Claude Mathieu avec la collaboration de Gail Pheterson, Les Cahiers du CEDREF (Université Paris 7), nº 7, 1998, 82 p.

Gail PHETERSON, The Prostitution Prism (Amsterdam University Press, 1996) : Le Prisme de la prostitution. Édition augmentée. Traduit de l’anglais (États- Unis) par NicoleClaude Mathieu. Paris : L’Harmattan, 2001 (coll. “Bibliothèque du féminisme”).

\section{Traducciones al español de textos de Nicole-Claude Mathieu}

(1997) "Relativismo cultural, ablación del clítoris y violencia contra las mujeres ". ARENAL, Revista de historia de las mujeres (Universidad de Granada), vol. 4, $\mathrm{n}^{\circ} 1$ (" Mujeres : Cuerpo e identidades"), janvier-juin, pp. 77-94. 
(2002) “Sexo y género”. En H. Hirata, F. Laborie, H. Le Doaré, D. Senotier (coordinadoras) Diccionario crítico del feminismo. Madrid : Sintesis , pp. 236-244.

(2005) "Identidad sexual/sexuada/de sexo ? Tres modos de conceptualización de la relación entre sexo y género ". En Curiel, Ochy \& Falquet, Jules (compiladoras) El Patriarcadao al desnudo. Tres feministas materialistas. Buenos Aires : Brecha lesbica, pp. 130-175. 